\title{
SISTEMATICIDAD Y UNIDAD DE LA EXPERIENCIA EN KANT
}

\section{PEDRO STEPANENKO}

INSTITUTO DE INVESTIGACIONES FILOSÓFICAS Universidad NACIONAL AuTónoma de MÉxico

Los grandes proyectos filosóficos suelen tener carencias, cuyo remedio pone en riesgo la estructura en la que descansan, como si llevaran en sí el germen que los puede destruir. Algunas veces son los propios autores de semejantes proyectos los que, al intentar reacomodar las piezas que han ensamblado, inician su descomposición. Quien se dedica actualmente a la historia de la filosofía puede sentirse atraído por esta imagen y abordar estos proyectos como el historiador que narra el surgimiento y la desaparición de una cultura. En el caso de Kant, uno puede ceder a esta tentación al tratar de explicar los cambios epistemológicos introducidos en el apéndice a la Dialéctica trascendental de la Crítica de la razón pura y confirmados en la Crítica del Juicio. En efecto, en estos textos, Kant le otorga a la idea de sistematicidad un lugar tan importante en el proceso del conocimiento que parece estar desarmando el andamiaje construido en la Analítica trascendental de la Crítica de la razón pura.

La acepción más amplia del término "sistema" significa, para Kant, simplemente un conjunto de conocimientos articulados bajo una sola unidad. En este sentido, la unidad de la experiencia, que Kant caracteriza en la Crítica de la razón pura como la unidad que conforman los principios trascendentales, es un sistema: ${ }^{1}$ en tanto que estos principios determinan lo que es un objeto en general articulan los conocimientos empíricos bajo una sola unidad. Conforme a ellos se estructura la experiencia de tal manera que podemos elaborar juicios acerca de objetos, juicios que pueden verificarse o refutarse en la experiencia. Estos principios son trascendentales porque

i Cfr. Primera introducción a la Crítica del del Juicio, 4a. sec. / Kants Gesammelte Schriften, Preußischen Akademie der Wissenschaften (en adelante: A.A.), vol. XX, pp. 208-209/ Primera introducción a la "Crítica del Juicio", trad. J.L. Zalabardo, Visor, Madrid, 1987 (en adelante: trad. Zalabardo), pp. 43-44. 
establecen las condiciones a priori- de posibilidad de la experiencia y son constitutivos en tanto que determinan lo que puede considerarse como un objeto de la experiencia. $^{2}$

En contraste con estos principios, expuestos en la Analítica trascendental, Kant introduce en un apéndice de la Dialéctica trascendental principios regulativos, que conforman una "unidad sistemática" con características muy distintas a la unidad de la experiencia en general. Se trata de principios que postulan $^{3}$ a priori un orden jerárquico entre leyes y conceptos empíricos, es decir, un orden bajo el cual podemos derivar unas leyes de otras, así como construir una pirámide de conceptos empíricos conforme a géneros y especies. Se trata, pues, de un plan que debemos ir descubriendo en la experiencia y que, sin embargo, presuponemos a priori o "proyectamos" sobre la naturaleza para guiar el descubrimiento de leyes y la formación de conceptos empíricos. Esto es lo que Kant llama "unidad sistemática" en sentido estricto y su principal característica, en contraste con la unidad de ta experiencia, es que nunca se cumple cabalmente, sino sólo gradualmente, conforme enriquecemos nuestros conocimientos empíricos y los vamos ajustando a esa unidad, que permanece siempre como un ideal (A 664). Lo que no queda claro en este apéndice es qué tan indispensables son estos principios para el desarrollo de la experiencia. Al comienzo de esta controvertida parte de la Crítica de la razón pura, Kant considera que esta unidad sistemática es hipotética, en la medida en que "no se afirma que tengamos que encontrarla de hecho, sino que [... ] hay que buscarla en beneficio de la razón" (A 649). ${ }^{4}$ Hay que buscarla para ordenar los conocimientos que ya hemos obtenido previanente. De esta manera, los principios regulativos de los cuales depende esta unidad parecen agregar algo nuevo al trabajo que el entendimiento ya ha realizado en colaboración con la sensibilidad y, por ello, no puede decirse que sean necesarios para el conocimiento de objetos de la experiencia. Según esta perspectiva, los principios constitutivos y las intuiciones serían suficientes para dar razón de la experiencia y, por lo tanto, de los conocimientos acerca de objetos y eventos que comprende la experiencia. La razón en su uso hipotético no condiciona, pues, el conocimiento de objetos y eventos aislados, sino que hace posible conocimientos ordenados bajo un sistema; un sistema en el cual los eventos se contemplan como sometidos a leyes empíricas y los objetos como pertenecientes a una jerarquía de géneros y especies.

2 Con respecto a esta caracterización de los principios constitutivos, véase Körner, $S$., "Ontologische Notwendigkeit und Begründung ontologischer Prinzipien" en Neue Hefte für Philosophie, no. 14, Vandenhoek \& Ruprecht, Gotinga, 1978, p. 13.

3 Kant utiliza el término "postular" con respecto a los principios regulativos en A 650 .

4 Traducción de P. Ribas, Alfaguara, Madrid, 1978 (en adelante: trad. Ribas), p. 535. 
Esta visión que ofrece el comienzo del mencionado apéndice apoya claramente la interpretación que Gerd Buchdahl ha proporcionado acerca de la unidad sistemática de la razón. ${ }^{5}$ Conforme a esta interpretación, en la filosofía de Kant hay que distinguir la experiencia, que opera simplemente bajo los principios constitutivos, de los conocimientos científicos, que requieren además la proyección de esa unidad sistemática. En el caso de la experiencia en general sólo pueden considerarse necesarios los principios constitutivos que condicionan la referencia a objetos y eventos particulares. Los conocimientos científicos, en cambio, pueden generar conocimientos empíricos necesarios, en la medida en que se integran a la unidad sistemática que la razón postula a priori. Ésta es una interpretación atractiva y sencilla; además, permite dar razón del cambio de teorías científicas desde la perspectiva kantiana, aun cuando la explicación de ese cambio no haya sido uno de los objetivos de Kant.

Pero el asunto se complica en el propio apéndice de la Crítica de la razón pura, cuando Kant aclara que el uso hipotético de la razón, por el cual integramos nuestros conceptos y conocimientos a la unidad sistemática, descansa en un principio trascendental, que postula esa unidad en los fenómenos de los cuales podemos obtener conceptos y leyes ordenados sistemáticamente. Se trata de un principio trascendental, porque exige la unidad racional, ya no de nuestros conocimientos, sino de los objetos mismos que podemos conocer. En él se apoya el uso hipotético de la razón, ya que, según explica Kant, sin presuponer homogeneidad en los fenómenos sería imposible obtener conceptos de esos fenómenos.

Si la diferencia [... entre los fenómenos que se nos ofrecen fuera tan grande, que el más agudo entendimiento humano fuera incapaz de encontrar la menor semejanza al compararlos entre sí (un caso que es perfectamente imaginable), entonces no existiría la ley lógica de los géneros, como no habría tampoco concepto alguno de género ni conceptos universales. Es más, no habria ni entendimiento, puesto que el único quehacer de éste son tales conceptos. (A 653-654. $)^{6}$

Conforme a este pasaje, la unidad sistemática es necesaria para que el entendimiento pueda crear conceptos empíricos. Si la razón no presupone $a$ $\nabla$ priori cierta homogeneidad entre los fenómenos, el entendimiento se queda

5 Cfr. Buchdahl, G., "The Kantian 'Dynamic of Reason' with Special Reference to the Place of Causality in Kant's System", en Beck, L.W. (ed.), Kant Studies Today, LaSalle, Illinois, Open Court, 1969; Metaphysics and the Philosophy of Science, Basil Blackwell, Oxford, 1969; "The Conception of Lawlikeness in Kant's Philosophy of Science", en Beck, L.W. (ed), Kant's Theory of Knowledge, Dordrecht, Reidel, 1974.

6 Trad. Ribas, p. 537. 
desorientado en medio de una multiplicidad que no le permite crear conceptos bajo los cuales caiga una diversidad de objetos. El entendimiento, sin la asistencia de esta unidad, sólo puede establecer semejanzas formales entre fenómenos; en cuanto al contenido no puede anticipar nada y, por ello, no puede guiar su propio trabajo comparativo de manera que genere conceptos empíricos. Kant incluso va más allá al sostener, en la última oración del pasaje citado, que sin esta unidad ni siquiera habría entendimiento. ¿Por qué llega tan lejos? La respuesta a esta pregunta me parece obvia: sería absurdo afirmar que el entendimiento puede conocer objetos de la experiencia sin hacer uso de conceptos empíricos. ${ }^{7}$ Debemos, entonces, pensar la aplicación de los principios constitutivos del entendimiento como una aplicación condicionada por la proyección de la unidad sistemática, sin la cual no es posible obtener conceptos empíricos. Así pues, de acuerdo con esta segunda perspectiva adoptada por Kant en el apéndice comentado, la unidad sistemática no sólo es un presupuesto del conocimiento científico, sino del conocimiento en general y, por lo tanto, es necesaria para aplicar el entendimiento a los datos de los sentidos.

En este misino apéndice, Kant presenta otra razón más para considerar a la unidad sistemática como necesaria para el conocimiento en general, a saber: que sin ella no tendríamos criterios de verdad empírica. Los principios constitutivos del entendimiento no son suficientes para proporcionar criterios de verdad, porque sólo señalan las características que debe tener todo fenómeno posible de la experiencia. Para tener un criterio de verdad no basta con determinar lo que es posible; necesitamos algo que nos permita seleccionar lo que es real entre lo que es posible. La unidad sistemática es lo que permite esta selección; ofrece un criterio para elegir entre todos los sucesos posibles. Sin este criterio el entendimiento también se perdería en un mar de fenómenos posibles.

La ley racional que dirige la búsqueda de tal unidad posee carácter necesario, pues, a falta de esa ley, careceríamos de razón y, sin ésta, no habría ningún uso coherente del entendimiento y, en ausencia de éste uso, no tendríamos criterio alguno suficiente de verdad empírica; en orden a este criterio, nos vemos, por tanto, obligados a dar por supuesto que esa unidad sistemática de la naturaleza es necesaria y posee plena validez objetiva. (A 651.) ${ }^{8}$

7 ¿Cabría pensar que los conceptos puros del entendimiento, aplicados directamente a sensaciones, proporcionan conocimiento de objetos sin necesidad de conceptos empíricos? Yo creo que Kant nunca sostuvo semejante extravagancia. En la deducción trascendental, particularmente en la primera edición de la Crítica de la razón pura, queda claro que las categorías son aquello que le proporciona objetividad a los conceptos empíricos, los cuales sintetizan una pluralidad específica de intuiciones empíricas.

8 Trad. Ribas, p. 536. 
Dos son, pues, las razones por las cuales Kant sostiene en el apéndice de la Crítica de la razón pura que la unidad sistemática es necesaria: porque permite la formación de conceptos empíricos y porque proporciona criterios de verdad empirica. Sin embargo, el propio apéndice es confuso y su posición en la Crítica de la razón pura es secundaria, por lo cual sería aventurado concluir que Kant esté transformando su concepción del conocimiento expuesto en el resto de la obra. La Crítica del Juicio, en cambio, presenta en la introducción misma una concepción del conocimiento semejante a la última de las perspectivas desarrolladas en el apéndice, es decir, una concepción para la cual la unidad sistemática es imprescindible para el conocimiento en general. Sólo que la postulación de la unidad sistemática aquí es reasignada al juicio reflexionante, entendido como la capacidad de generar conceptos generales mediante reflexión a partir de objetos particulares. Como Paul Guyer lo muestra en su artículo "The Principles of Reflective Judgement", las cracterísticas de los principios regulativos son reasignadas al principio del juicio reflexionante. Se trata, pues, de un principio que presupone a priori una sistematicidad en la naturaleza, gracias al cual es posible ordenar nuestros conceptos empíricos y no perdernos en la infinita multiplicidad de conocimientos posibles que los principios constitutivos deja abierta. Igualmente, se trata de un principio que, a pesar de postular a priori la sistematicidad en la naturaleza, sólo se cumple gradualmente, conforme vamos integrando una mayor diversidad de fenómenos a un menor número de leyes empíricas.

Pero, en la filosofía de Kant, afirmar la necesidad de un principio del juicio equivale a condicionar a este principio la aplicación de los principios constitutivos del entendimiento a los datos que proporciona la sensibilidad en la experiencia. El juicio es una facultad que Kant ubica entre el entendimiento y la sensibilidad; es la capacidad de subsumir intuiciones bajo conceptos que ya tenemos o la capacidad de buscar conceptos bajo los cuales pueden caer intuiciones dadas. Si el juicio posee un principio autónomo y necesario para la experiencia, entonces el entendimiento debe ponerse en contacto con los datos de los sentidos condicionado por este principio. Esto parece borrrar la diferencia entre los principios constitutivos y el principio que postula la unidad sistemática en la naturaleza; parece hacer confluir el trabajo del entendimiento con el del juicio, de tal manera que habría que homogeneizar el carácter de sus principios. Esta homogeneización puede pensarse de dos maneras. Puede pensarse que el principio del juicio reflexionante en realidad debe ser un principio constitutivo, en la medida en que condiciona la aplicación de los principios constitutivos del entendimiento. $O$ bien, puede pensarse que los principios constitutivos del entendimiento deben transformarse en un ideal, ya que su aplica- 
ción se da a través de un principio que sólo se cumple gradualmente. La primera posibilidad la rechaza Kant en la propia introducción a la Crítica del Juicio, en donde insiste en que la sistematicidad de conceptos y leyes empíricas no es una condición necesaria de los objetos de la experiencia, puesto que es contingente desde la perspectiva de los principios constitutivos, aquellos principios que determinan a priori qué puede considerarse como un objeto de la naturaleza o una regularidad atribuible a la misma. ${ }^{9}$ Pero la segunda posibilidad podría tomarse como una sugerencia para hacer concebible que la sistematicidad sea una condición de la aplicación de los principios constitutivos, a saber: considerar que la unidad que estos últimos constituyen, la unidad de la experiencia, es un principio regulativo, es decir, un principio cuya satisfacción es gradual, sin que sea posible alcanzar su plena satisfacción. En su artículo "Reason and Reflective Judgement", Paul Guyer plantea precisamente esta alternativa, sin decidirse finalmente a sostenerla.

If the concept of sistemacity is needed to complete the application of the categories to empirical intuition and thereby constitute the unity of experience, yet remains more an open-ended task than a condition which can ever be completely satisfed in intuition, then must not the unity of experience itself also become a regulative ideal rather than a constitutive concept? ${ }^{10}$

Que la unidad de la experiencia deje de ser un elemento constitutivo y se convierta en un ideal regulativo es una transformación radical de la concepción del conocimiento expuesta en la Crítica de la razón pura. Semejante transformación significaría que las condiciones de objetividad de nuestros conocimientos sólo se cumplen gradualmente e incluso que podrían variar de acuerdo con la sistematicidad particular que en cada momento vayamos descubriendo entre los fenómenos. Esto haría imposible el proyecto que Kant trata de realizar en la Analítica trascendental, es decir, una metafísica que establezca lo que podemos saber a priori de los objetos de la experiencia.

Ralph Walker sostiene en su artículo "Kant's Conception of Empirical Law"11 que los cambios en la concepción del conocimiento que ofrece la introducción a la Crítica del Juicio se deben a que Kant vuelve a plantearse el problema de la conformidad de los principios que gobiernan el pensamiento con el mundo; pero esta vez, a diferencia de la Crítica de la razón pura, reconoce el problema de la inducción. Para Walker, el requerimiento de la unidad sistemática de nuestros conocimientos en realidad no es más que un

9 Cfr. Introducción a la Crítica del Juicio, 5a. parte / A.A., V, p. 184 / trad. M.G. Morente, Editora Nacional, México, D.F, 1975 (en adelante: trad. Morente), p. 133.

10 En Noûs, no. 24, 1990, p. 19.

11 En The Aristotelian Society, volumen suplementario LXVI, 1990, pp. 243-258. 
disfraz del problema de la inducción y ofrece una explicación alternativa a la necesidad que le adjudicamos a ciertos conocimientos, dejando de lado la explicación que ofrece Kant en la primera Crítica. Mientras que en la Crítica de la razón pura, Kant recurre al idealismo trascendental para dar cuenta de esa necesidad, en la Crítica del Juicio apela a la idea de sistematicidad. Una ley de la naturaleza, conforme a esta última obra, es considerada necesaria cuando concuerda con un sistema de leyes, gracias al cual podemos explicar una diversidad de fenómenos, y no por derivarse de ciertas estructuras a priori que supuestamente posee el sujeto del conocimiento. ¿Cabe, pues, sospechar que Kant se ve obligado en la Crítica del Juicio a transformar radicalmente sus ideas expuestas en la primera Crítica debido a que, por primera vez, se enfrenta seriamente al problema de la inducción?

Yo creo que la respuesta correcta a esta pregunta es negativa, así como lo es también la respuesta al problema que plantea Paul Guyer en el artículo antes mencionado. Es decir, creo que es posible aceptar las ideas desarrolladas en la tercera Crítica sobre el juicio reflexionante sin tener que transformar la unidad de la experiencia en un ideal regulativo y que reconocer el peso que tiene la inducción en el proceso del conocimiento no quebranta la idea de Kant acerca de los principios constitutivos o condiciones de posibilidad de la experiencia. En la siguiente sección de este artículo intentaré mostrar que el juicio reflexionante responde a preocupaciones distintas de aquellas a las que responde la unidad de la experiencia en general y que, por lo tanto, la tensión que se ha querido ver entre ellos es sólo aparente.

II

En la exposición que Kant ofrece del juicio reflexionante en la introducción a la Crítica del Juicio es posible distinguir dos funciones de este tipo de juicio. La primera consiste en guiarnos entre diversas explicaciones posibles de los mismos fenómenos o entre distintas leyes empíricas posibles, según su integración a un sistema. La segunda permite formar conceptos empíricos en general bajo el supuesto de una estructura en la cual deben encajar. Ambas funciones se apoyan en un principio, según el cual debemos presuponer que la naturaleza es un sistema, sin poder determinar a priori la forma específica de ese sistema.

Uno de los pasajes en donde más claramente queda expuesta la primera función del juicio reflexionante, en contraste con los principios constitutivos del entendimiento, es el siguiente:

Encontramos en las bases de la posibilidad de una experiencia, primero, sin duda, algo necesario, a saber: las leyes generales, sin las cuales la naturaleza en general (como objeto de los sentidos) no puede ser pensada, y éstas des- 
cansan en las categorías, aplicadas a las condiciones formales de toda intuición posible para nosotros, en cuanto ésta es dada también a priori. Ahora bien: bajo esas leyes es determinante el Juicio, pues no tiene más que subsumir bajo leyes dadas. Por ejemplo, dice el entendimiento: Todo cambio tiene su causa (ley general de la naturaleza): el Juicio trascendental no tiene ahora más que indicar a priori la condición de la subsunción bajo el concepto dado del entendimiento, y ésta es la sucesión de las determinaciones de una y la misma cosa. Para la naturaleza, empero, en general (como objeto de experiencia posible), aquella ley es conocida como absolutamente necesaria. Pero [...] los objetos del conocimiento empírico, aparte de aquella condición formal del tiempo, son además determinados o, en cuanto se puede juzgar a priori, determinables de diferentes modos; así que naturalezas específicamente distintas, aparte de lo que tengan en común, como pertenecientes en general a la naturaleza, pueden ser causas en maneras infinitamente diversas, y cada una de estas maneras debe (según el concepto de una causa en general) tener su regla, la cual es ley [... ] Por eso el Juicio debe, para su propio uso, aceptar como principio a priori que lo contingente para la humana investigación en las leyes particulares (empíricas) de la naturaleza encierra una unidad en el enlace de su diversidad con una experiencia posible en sí, unidad que nosotros no tenemos ciertamente que fundar, pero pensable, sin embargo, y conforme a la ley. ${ }^{12}$

De acuerdo con este pasaje, las leyes necesarias del entendimiento, aquellos principios que establecen lo que puede ser pensado como parte de la experiencia o de la naturaleza en general, aceptan una gran diversidad con respecto a los modos más específicos en que pueden determinarse los objetos del conocimiento. Estas leyes necesarias son los principios constitutivos que se obtienen de las categorías kantianas. Ya que estos principios aceptan una diversidad prácticamente infinita de fenómenos posibles, se requiere otro principio que nos permita orientar la investigación en la búsqueda de regularidades específicas entre fenómenos o leyes particulares empíricas. Este principio debe postular un orden particular dentro del ámbito de posibilidades que abren las categorías, aun cuando no podamos especificar este orden sin la ayuda de los contenidos específicos de la experiencia. A este orden particular indeterminado lo llama Kant "sistematicidad" y el principio que lo postula es el principio del juicio reflexionante.

La introducción de este principio creo que puede interpretarse de dos maneras distintas. Puede tratarse tan sólo de un principio que reduzca el ámbito de posibilidades que aceptan las categorías o bien puede ser un principio que nos permita elegir entre varias explicaciones posibles de los mismos fenómenos. En el primer caso, el principio del juicio reflexionante recorta el espacio de los fenómenos posibles, de tal manera que constituya

12 Introducción a la Crítica del Juicio, 5a. sec. / A.A., V, pp. 182-184 / trad. Morente, pp. 129-131. 
una unidad abarcable de leyes empíricas para el entendimiento humano. De acuerdo con esta versión, destaca el aspecto heurístico de presuponer sistematicidad en los conocimientos empíricos, ya que está claro que recortar el ámbito de posibilidades no representa una determinación objetiva de los fenómenos, sino que responde a un principio con valor subjetivo, a una máxima que nos ayuda a descubrir clases de fenómenos y leyes empíricas. ${ }^{13}$ De acuerdo con la segunda interpretación, el principio del juicio reflexionante guía el descubrimiento de regularidades y leyes empíricas en un sentido más fuerte, en la medida en que nos permite elegir una entre varias explicaciones posibles de los fenómenos. En este caso, creo que las explicaciones, de las cuales hay que elegir una, debemos pensarlas como productos sólo del entendimiento. Esto significa que el juicio reflexionante interviene una vez que el entendimiento ya ha presentado, por su cuenta, las explicaciones posibles, es decir, las explicaciones que cumplen con las condiciones de objetividad que establecen los principios derivados de las categorías. El juicio reflexionante, entonces, escoge la explicación que mejor se adapta a un sistema de leyes empíricas y determina así la explicación que debemos considerar correcta. En este sentido, el principio del juicio reflexionante, aquél bajo el cual suponemos que la naturaleza es un sistema de leyes empíricas, funciona como criterio de verdad, en contraste con los criterios de objetividad que establecen los principios constitutivos. Estos últimos seleccionan las explicaciones que pueden ser verificadas o refutadas en la experiencia, ya que determinan, por ejemplo, relaciones de causalidad y atribución que pueden corresponder a lo que nos puede ser dado en la experiencia. Pero puede haber muchas explicaciones de los mismos fenómenos que cumplen con estos requisitos y el entendimiento solo ya no es capaz de señalar a cuál de ellas debemos asentir. El juicio reflexionante, mediante la idea de sistematicidad, suple precisamente esta carencia y proporciona, de esta manera, un criterio de verdad, en tanto que nos ayuda a decidir a cuál de las explicaciones debemos asentir.

Creo que una de las razones por las cuales parece haber tensión entre los principios constitutivos y el principio del juicio reflexionante creo que se debe a que no se presta suficiente atención a esta diferencia de funciones. Con frecuencia se considera que los principios constitutivos, expuestos en la Analítica trascendental de la Crítica de la razón pura, proporcionan criterios de verdad. ${ }^{14}$ De ser asi, efectivamente tendríamos un conflicto entre

13 Cfr. Ibid. / A.A., V, p. 184 / trad. Morente, p. 132.

14 Véase Kitcher, Philip, "Proyecting the Order of Nature", en Butts, R.E. (ed.), Kant's Philosophy of Physical Science, D. Reidel, Dordrecht, 1986, p. 207. También: Walker, Ralph., "Kant's Conception of Empirical Law", en The Aristotelian Society, volumen suplementario LXVI, 1990, p. 245 / Coherence Theory of Truth, Routledge, Londres, 1989, cap. 4. 
los criterios que se derivan de las categorías y el que se deriva del juicio reflexionante. Por un lado, habría criterios que establecerían el valor de verdad de enunciados empíricos en forma aislada, caso por caso; por el otro, habría criterios que exigirían la integración de esos enunciados a un sistema de conocimientos para poder decidir su valor de verdad. Tendríamos, por un lado, conocimientos empíricos aislados unos de otros, que dependerían sólo de la caracterización general de lo que es un objeto de la experiencia $\mathrm{y}$, por el otro, conocimientos empíricos, cuya verdad sería relativa a un sistema de conocimientos empíricos. Esta diferencia de criterios de verdad sería aceptable, si se distinguieran dos tipos de conocimientos empíricos. La primera caracterización que Kant presenta en el apéndice a la Diálectica trascendental podría estar sugiriendo una distinción de este tipo. Pero en la Crítica del Juicio, Kant hace intervenir los principios constitutivos y la idea de sistematicidad en un mismo proceso de generación de conocimientos, aunque en distintos momentos o aspectos de ese proceso. Si se interpretan, pues, los principios constitutivos y el juicio reflexionante como si ambos ofrecieran, ambos, criterios de verdad, nos enfrentaríamos, en el mejor de los casos, a una tensión que no creo que pueda resolverse. Sin embargo, esta tensión desaparece si se comprenden los principios constitutivos sólo como criterios de objetividad, es decir, como criterios que definen qué enunciados pueden tener un valor de verdad determinable en la experiencia. $Y$ esta comprensión, me parece, es la que Kant ofrece desde la introducción a la Analítica trascendental de la Crítica de la razón pura. ${ }^{15}$

La diferencia entre criterios de objetividad y criterios de verdad creo que evita una concepción del juicio reflexionante que parezca quebrantar los principios constitutivos. Gracias a esta diferencia, los principios constitutivos se pueden concebir como aquello que determina el tablero de posibilidades dentro del cual opera el juicio reflexionante. Pero esta solución sólo le concierne a la primera de las funciones del juicio reflexionante que he mencionado al comienzo de esta sección. Salvar la tensión entre los principios constitutivos y la segunda función del juicio reflexionante puede resultar más complicado, puesto que esta función consiste en obtener los conceptos empíricos sin los cuales no sería posible aplicar los principios constitutivos a los elementos que nos proporciona la sensibilidad en la experiencia. Si el proceso mediante el cual obtenemos conceptos empíricos se apoya en un principio que presupone sistematicidad en la naturaleza y,

15 Es cierto que Kant, en esta introducción, utiliza el término "verdad" para referirse al valor de verdad. Yo creo que esto ha propiciado la confusión entre criterios de verdad y criterios de objetividad. Con respecto a ese uso del término "verdad" en Kant, véase el excelente artículo de Gerold Prauss, “Zum Wahrheitsproblem bei Kant" en Kant-Studien, no. 60, 1969; en especial la p. 179. 
además, este principio sólo se cumple gradualmente, conforme nuestros conocimientos muestran una mayor sistematicidad, entonces, parece natural concluir que todos los principios que se aplican a la sensibilidad usando conceptos empíricos sólo se cumplen gradualmente.

La definición misma que Kant proporciona del juicio reflexionante, en la primera introducción a la Crítica del Juicio, sugiere que debemos presuponer sistematicidad en la naturaleza para poder generar conceptos empíricos en general. El juicio reflexionante, conforme a esta introducción, es la "facultad de reflexionar sobre una representación dada según cierto principio, para llegar a un concepto hecho posible por aquélla". ${ }^{16}$ Ese principio lo formula Kant en unas líneas más abajo, de la siguiente manera:

El principio de la reflexión sobre objetos dados de la naturaleza es que se puedan encontrar conceptos determinados empíricamente para todas las cosas de la naturaleza, en otras palabras, que siempre se puede presuponer en sus productos una forma que sea posible según leyes generales cognoscibles por nosotros. Porque si no pudiéramos presuponer esto y no pusiéramos este principio como fundamento de nuestro tratamiento de las representaciones empíricas, toda la reflexión se realizaría azarosa y ciegamente, y por ello sin ninguna esperanza sólida de encontrar su concordancia con la naturaleza. ${ }^{17}$

Esta formulación del principio del juicio reflexionante deja claro que presuponer a la naturaleza como un sistema de leyes y formas naturales no sólo es necesario para ordenar los conceptos empíricos de acuerdo con géneros y especies, sino en general para obtener cualquier concepto de un objeto de la naturaleza. Si pensamos que la Analítica trascendental de la Crítica de la razón pura comprende una explicación de cómo obtenemos conceptos empíricos, entonces vuelve a surgir un conflicto entre la concepción del conocimiento que ofrece esta parte central de la primera Crítica y la que Kant propone en la Crítica del Juicio. Semejante explicación apelaría exclusivamente al entendimiento y a la sensibilidad, aceptando que podemos generar conceptos empíricos sin presuponer ningún sistema de leyes empíricas y formas naturales. Quizá algunos pasajes sugieran una explicación de este tipo, pero el proyecto de la Analítica no contempla una explicación del proceso de formación de conceptos empíricos. La Analítica trascendental pretende establecer los conceptos y los principios que garantizan la objetividad de nuestros conocimientos, no explicar cómo obtenemos conceptos a partir de lo que nos es dado en la experiencia. Para evitar la tensión entre categorías y principios constitutivos, por un lado, y el juicio reflexionante, por el otro,

16 Primera introducción a la Crítica de juicio, 5a. sec. / A.A., vol. XX, p. 211 / trad. Zalabardo, p. 49.

17 Ibid. 
en este caso también hay que enfatizar que los primeros se limitan a garantizar la objetividad de nuestras representaciones. Con respecto a la primera de las funciones del juicio reflexionante, la solución consistía en señalar que los principios constitutivos determinan el campo de los enunciados que pueden ser verificados o refutados en la experiencia, es decir, el ámbito de los enunciados objetivos. Con respecto a la segunda función, que tiene que ver con la formación de conceptos empíricos, la solución creo que consiste en señalar que las categorías son los conceptos que determinan lo que es un objeto de la experiencia en general y, por lo tanto, que son los conceptos que nos permiten evaluar si bajo cualquier otro concepto pueden caer objetos de la experiencia, es decir, si puede tener referencia. En la Crítica de la razón pura, Kant afirma con claridad que las categorías son lo que garantiza la objetividad de los conceptos empíricos (A 109); esto significa que son ellas las que establecen las condiciones que debe cumplir un concepto para que represente objetos posibles de la experiencia. Si un concepto empírico, por ejemplo, funciona para sintetizar intuiciones de acuerdo con las categorías de sustancia y de causa, entonces es objetivo. Si el concepto de un objeto no nos permite establecer relaciones de causalidad entre ese supuesto objeto y otros objetos posibles, entonces carece de objetividad. Si además ese concepto pertenece a un sistema más específico, es algo que las categorías no toman en consideración. Si el concepto se ha obtenido presuponiendo que la naturaleza es un sistema de formas y leyes empíricas, tampoco es algo que las categorías deban tener presente para evaluar su validez objetiva. Puede hablarse de criterios de objetividad más específicos, pero éstos deben ceñirse a los criterios necesarios de objetividad que ellas establecen. Puede incluso decirse que estos criterios más específicos son necesarios para obtener los conceptos empíricos que las categorías evalúan, pero eso no tiene por qué hacer variar su evaluación, ni tiene por qué convertitrla en un proceso que acepte distintos grados de cumplimiento.

La diferencia entre la quid iuris y la quid facti, que Kant introduce al inicio de la deducción trascendental de las categorías, en la primera Crítica (A 84-92), creo que puede ayudarnos a ver las diferentes funciones que desempeñan, por un lado, las categorías y, por el otro, el juicio reflexionante. La quid iuris responde a la pregunta por el derecho que tenemos al considerar un concepto como objetivo. La quid facti responde a la pregunta por la manera en que hemos obtenido un concepto. Las categorías constituyen la respuesta de Kant a la pregunta por la quid iuris; el juicio reflexionante, que presupone sistematicidad en la naturaleza para obtener conceptos empíricos, creo que puede contribuir a responder la quid facti. De ser así, la sistematicidad por sí misma no podría considerarse como 
garantía de objetividad, sólo podría convertirse en criterio de objetividad en el interior del marco que establecen las categorías.

Es cierto que Kant afirma en la introducción a la Crítica del Juicio que las máximas del juicio reflexionante no tienen un origen psicológico, ${ }^{18}$ lo cual parece hablar en contra de clasificarlas como elementos que contribuyen a responder la quid facti. La razón de esta afirmación es que estas máximas no describen cómo operan nuestras facultades, sino que establecen lo que debemos presuponer para realizar determinada operación. En el pasaje en el cual advierte lo anterior, Kant se refiere sólo a lo que he llamado la primera función del juicio reflexionante. Pero también podría aceptarse esto mismo para los principios que hacen posible la formación de conceptos empíricos y, asi, habría que decir que Kant no se interesa por describir los procesos mediante los cuales adquirimos esos conceptos, sino por determinar lo que necesitamos presuponer acerca de la naturaleza para poder adquirirlos. Aún aceptando que Kant se interese sólo por esto último, me parece que hay una clara diferencia entre lo que necesitamos presuponer para adquirir conceptos empíricos y lo que necesitamos presuponer para considerar que esos mismos conceptos sean objetivos. Y esta diferencia es la que evita el conflicto entre los principios constitutivos y el juicio reflexionante. De los primeros se puede exigir que se cumplan cabalmente, sin por ello entrar en conflicto con el carácter ideal de la sistematicidad que presupone el juicio reflexionante.

Reconocer que la filosofía de Kant hace intervenir distintos tipos de principios en la explicación del conocimiento es la clave para disolver los conflictos que parecen surgir al confrontar la Crítica de la razón pura con la Crítica del Juicio. Creo que las diferencias que he tratado de enfatizar creo que contribuyen a ofrecer una visión más armónica y compleja de la teoría del conocimiento kantiana que aquella que se limita a enfatizar los conflictos. Pero resulta difícil reconocer estas diferencias cuando se quiere ver en las categorías y en los principios constitutivos los conceptos y las leyes más generales de un sistema particular. Un sistema que sólo podría completarse gradualmente a través del juicio reflexionante, el cual proporcionaría las leyes y conceptos intermedios, que harían posible conectar aquellos conceptos y leyes con los datos particulares de los sentidos. L̀a consecuencia de semejante interpretación es clara: si el sistema completo es un ideal, los conceptos y leyes más generales del mismo también deben cumplirse sólo gradualmente. Pero Kant excluye esta interpretación cuando afirma que al juicio reflexionante le "incumbe poner las leyes particulares - también según lo que tienen de diferente bajo las mismas leyes generales

18 5a. sec. / A.A., V, p. $182 / \operatorname{trad}$. Morente, pp. 128-129. 
de la naturaleza - bajo leyes superiores, aunque todavía empíricas [.. . ]"19 Este pasaje deja claro que, para Kant, las leyes más generales del sistema que puede constituir la naturaleza son empírircas y que todas las leyes del sistema, por igual, caen bajo los principios constitutivos. Esto significa que no debemos identificar los principios constitutivos con las leyes más generales de un sistema, sino con los principios sobre los cuales descansa el sistema entero de leyes empíricas. Puede haber varios motivos para querer ver en los principios constitutivos las leyes más generales de un sistema; uno de ellos puede ser el compromiso que Kant establece entre su filosofía y la ciencia de su época. Pero uno de los motivos que propicia esta confusión, según creo, es la ambigüedad del término "unidad de la experiencia", que no es más que un caso particular de la ambigüedad con la que Kant usa el término "unidad". Este término puede significar el resultado de una síntesis o puede querer decir el campo dentro del cual se dan las síntesis que proporcionan conocimiento de objetos. Si se entiende por "unidad de la experiencia" el resultado de una síntesis, cuyos rasgos más generales están expresados por los principios constitutivos, está claro que se piensa en una unidad cambiante y cada vez más amplia. Sin embargo, no es en este sentido en el que Kant sostiene que los principios constitutivos conforman la unidad de la experiencia, sino en el segundo, es decir, en tanto que constituyen el marco dentro del cual se da el conocimiento empírico, el marco dentro del cual el conocimiento puede trazar distintas figuras con distintas magnitudes y gracias al cual puede hablarse de cambio de nuestros conocimientos.

Creo, pues, que si se reconocen las distintas funciones que desempeñan, por un lado, los principios constitutivos y, por el otro, el juicio reflexionante, entonces la unidad de la experiencia, que conforman los primeros, no tiene por qué perder su carácter necesario y convertirse en una tarea que sólo se cumple gradualmente; y esto incluso aceptando que los conceptos empíricos, que se requieren para la aplicación de aquellos principios a los datos de los sentidos, se obtienen presuponiendo el ideal de sistematicidad. No reconocer la diferencia entre estas dos funciones no sólo debilitaría el papel que cumplen las categorías en la filosofía de Kant, sino que, además, haría inexplicable la introducción misma del ideal de sistematicidad. Si las categorías se cumplieran sólo gradualmente, ellas mismas vendrían a desempeñar una de las principales tareas que Kant le otorga al ideal de sistematicidad. Entre varios conceptos o explicaciones posibles de los mismos fenómenos, sería posible elegir uno de ellos apelando tan sólo al ideal que las categorías proponen. Con ello, creo que se perdería la principal virtud que representa

19 Primera introducción a la Crítica del Juicio, 4a. sec. / A.A., XX, p. 210 / trad. Zalabardo, p. 44. 
introducir el ideal de sistematicidad en la filosofía kantiana, a saber: poder dar razón del cambio de nuestros conocimientos sin tener que modificar el esquema conceptual que nos permite reconocer, a pesar del cambio, que se refieren a los mismos objetos; hacer comprensible que distintas teorías o concepciones de la naturaleza pretendan explicar los mismos fenómenos. 